\title{
Chile y su política comercial "lateral"
}

\section{Sebastián Sáez \\ Ex Jefe del Depto. \\ ALCA-América del Norte, \\ Dirección General de \\ Relaciones Económicas \\ Internacionales, \\ Ministerio de Relaciones \\ Exteriores de Chile \\ Juan Gabriel Valdés S. Embajador, \\ Director General de Relaciones Económicas Internacionales, Ministerio de Relaciones \\ Exteriores de Chile}

En este artículo se examinan los fundamentos, objetivos y resultados de la política comercial "lateral" adoptada por Chile a partir de la década de 1990. En particular, se busca clarificar el papel de los acuerdos bilaterales e incorporar a la discusión la evidencia empírica observada en el caso de Chile. Se concluye que las críticas que se han formulado a esta política, especialmente por quienes privilegian la apertura unilateral por sobre otras opciones, se basan en un análisis limitado de los fundamentos teóricos del comercio internacional. Por ello, se argumenta, es importante ampliar los conceptos económicos considerados, para evaluar la racionalidad económica y política de esta estrategia, reconociendo la complementariedad que existe entre las opciones disponibles, e incorporando el análisis de teoría de juegos, la existencia de economías de escala, los costos de transacción existentes en el funcionamiento de los mercados internacionales, y elementos de política exterior. Con esta estrategia multidimensional Chile ha apuntado a superar diversos problemas y a estimular las actividades económicas más dinámicas que han caracterizado a su economía en los años noventa: exportaciones con mayor valor agregado, de servicios y de capitales. Los resultados obtenidos, medidos por los parámetros tradicionales de evaluación, no acusan efectos negativos y sí muestran efectos positivos. 


\section{I}

\section{Introducción}

Chile ha sido uno de los países latinoamericanos que han impulsado durante los años noventa una de las más activas políticas de acuerdos comerciales bilaterales, con fundamentos tanto de política exterior como económicos. Esta experiencia difiere de las políticas de integración impulsadas desde los años sesenta en tres aspectos: i) se basa en la suscripción de acuerdos de libre comercio de carácter amplio en el marco de una política de desarrollo hacia afuera y no en la creación de mercados comunes o uniones aduaneras; ii) los acuerdos han estimulado, con diverso grado de éxito, la inclusión de las distintas dimensiones del comercio, reflejando la mayor complejidad de las relaciones económicas internacionales de hoy, y iii) la búsqueda de acuerdos no se limita a los países de la región, aunque sí asigna prioridad a estos países —en especial a los de la Asociación Latinoamericana de Integración (ALADI) — , sino que se ha procurado avanzar también en la integración con los Estados Unidos y Canadá, con las economías del Asia-Pacífico y con la Unión Europea. Esto es un reconocimiento de la diversificación geográfica de los vínculos económicos de Chile.

La política indicada no ha estado exenta de críticas que se basan en diversos argumentos, fundamentalmente en el campo de las políticas comerciales. En yecto de rebaja de los aranceles desde un 11 a un 6\% en un período de cinco años, aprobado por el Congreso, un grupo de economistas de diversa inspiración política argumentó a favor de la propuesta de rebaja uniforme presentada por el gobierno; señalaban que ésta corregiría "importantes distorsiones del esquema arancelario vigente y le permitirá al país mejorar su inserción internacional", y agregaban que "la firma de un sinnúmero de acuerdos comerciales ha permitido que el arancel uniforme del $11 \%$ se haya transformaparticular, en el marco de la discusión acerca del pro- do en la práctica en un arancel altamente diferenciado, [que] resulta en protección efectiva negativa para algunos sectores y bastante mayor que el $11 \%$ para otros" (El Mercurio, 1998).

Esta discusión es semejante a la que tuvo lugar a comienzos de los años noventa acerca de qué tipo de política comercial debía seguir el Gobierno de los Estados Unidos para promover la apertura comercial. Lawrence Summers, actual Subsecretario del Tesoro de los Estados Unidos, señaló frente a esta polémica que debería haber una "presunción rebatible en favor de todas las reducciones laterales en las barreras comerciales, sean éstas multi, uni, tri, o plurilaterales' (Frankel, 1997). Con ello quería significar que esta discusión no tenía especial relevancia, sino que lo importante era aprovechar las oportunidades comerciales que se presentaran y que todas las iniciativas "laterales" eran buenas.

Subyacen el debate público chileno dos aspectos de interés: i) un número representativo de la profesión rechaza los aranceles diferenciados y sus efectos; y ii) se privilegia una política de apertura unilateral, por sobre otras opciones.

El propósito de este trabajo es exponer los fundamentos y objetivos de la política comercial "lateral" seguida por Chile desde 1990 a la fecha. Para hacerlo, se analiza el aspecto relacionado con la apertura unilateral, fundamentando las opciones de política impulsadas desde el advenimiento de la democracia en Chile. ${ }^{1}$ En particular, se intenta clarificar el papel que han desempeñado y desempeñan los acuerdos bilaterales en la política comercial de los gobiernos de la Concertación de manera de contribuir a una mejor comprensión de los objetivos perseguidos, que son económicos y políticos a la vez, e incorporar al debate la información empírica, aplicable al caso de Chile, existente hasta ahora sobre la materia. $\square$ Los autores agradecen los valiosos comentarios a una versión anterior de este artículo que hicieron Alicia Frohmann, Patricio Leiva, Raúl E. Sáez y funcionarios de la Dirección General de Relaciones Económicas Internacionales (DIRECON).

\footnotetext{
1 Véase un análisis del período del Presidente Patricio Aylwin en Sáez, Salazar y Vicuña, 1995.
} 


\section{II}

\section{El papel de los acuerdos económicos}

\section{y las opciones de política comercial}

Las principales conclusiones que la teoría económica entrega respecto de la política comercial señalan que, para un país pequeño como Chile, la apertura unilateral es la mejor política, ya que contribuye a una asignación de recursos más adecuada y, en consecuencia, maximiza el bienestar de la comunidad como un todo. Ha sido reconocido por economistas de distinta orientación política que la apertura unilateral aplicada por Chile desde mediados de los años setenta contribuyó al acelerado crecimiento de sus exportaciones tanto tradicionales como no tradicionales, y a la vez a estimular una mayor diversificación en términos de productos y mercados de destino. ${ }^{2}$ No menos importante fue el papel que desempeñó el Estado en los años sesenta y setenta en la creación de incentivos de distinta naturaleza que contribuyeron a disminuir el riesgo del negocio exportador y de esta manera a crear un proceso endógeno de desarrollo comercial. ${ }^{3}$

Los gobiernos de la Concertación han reconocido la validez de este argumento y han promovido y profundizado la apertura unilateral. En efecto, en 1991 los aranceles fueron reducidos de manera uniforme desde un 15 a un $11 \%$, y recientemente el Congreso ha aprobado una nueva disminución de 5 puntos porcentuales adicionales en un período de cinco años. De esta manera, el arancel uniforme aplicable a las importaciones provenientes de países con los cuales Chile no ha suscrito acuerdos de libre comercio será sólo de $6 \%$, el más bajo de las últimas cuatro décadas. ${ }^{4}$
Sin embargo, la Concertación ha concentrado los avances en la apertura en áreas que afectan el comercio desde otras dimensiones: en particular, en el sector de telecomunicaciones se promovió una amplia desregulación y se introdujo mayor competencia, y en materia financiera se amplió el ámbito de negocios, mejorando la supervisión bancaria, de seguros y valores. El sector privado ha comenzado a invertir en infraestructura pública. Finalmente, el proceso de privatizaciones de empresas públicas ha continuado, cuidando en particular la transparencia e interés del Estado en este proceso. En un área de tanta importancia como la propiedad intelectual, Chile se convirtió en 1991 en el primer país latinoamericano en otorgar protección a los productos farmacéuticos. Además, ha ratificado importantes tratados internacionales, como el Convenio de París en 1991 y el Convenio internacional para la protección de variedades vegetales en 1996. En materia de inversión extranjera ha suscrito numerosos acuerdos de promoción y protección de inversiones, dando una señal clara de la estabilidad de sus políticas.

En síntesis, el proceso de apertura impulsado por los gobiernos de la Concertación no puede ser evaluado exclusivamente a través de los parámetros tradicionales del comercio (reducción de aranceles y eliminación de medidas no arancelarias), sino que debe entenderse dentro del conjunto de elementos que conforman las nuevas dimensiones de la política comercial.

\section{III}

\section{El papel de los acuerdos económicos}

\footnotetext{
2 Véase, por ejemplo, Wisecarver (ed., 1992) y Meller (1996).

3 Véanse los comentarios de Meller (1996) y los trabajos de Sáez (1994a y b).

${ }^{4}$ Debemos agregar que el régimen comercial chileno se caracteriza por la inexistencia de barreras no arancelarias y otros tipos de mecanismos adicionales de protección (excepto para un número muy restringido de productos agrícolas). Si consideramos los acuerdos vigentes, el arancel será sustancialmente menor.
}

\section{1. ¿Qué papel han desempeñado los acuerdos económicos internacionales negociados por Chile?}

Los acuerdos económicos internacionales, multilaterales, plurilaterales y bilaterales, han complementado y completado la apertura unilateral en aquellos aspectos en que ésta no puede intervenir ni resolver. 
Esta aseveración se puede examinar a la luz del caso concreto de un producto de exportación no tradicional "menor": los ostiones frescos y congelados. A fines de la década de 1980, luego de importantes inversiones realizadas en el Norte Chico de Chile, comenzó el desarrollo de las exportaciones de ostiones. Este producto fue orientado en un 60 a $70 \%$ al mercado francés. Sin embargo, en 1993, el Gobierno de Francia decidió que los ostiones chilenos (y de otros orígenes) no eran similares a los franceses y estableció un requisito de etiquetado y comercialización que exigía que en el mercado francés sólo podían venderse como «coquille Saint-Jacques» los ostiones de la especie Pecten. El resto, entre los que figuraba el producto chileno, debía venderse como "pétoncle», que el consumidor francés asociaba con un producto de menor calidad y precio. Esta clasificación "científica" afectó artificialmente las condiciones de competencia, produciendo una protección encubierta en favor del producto francés, que mantuvo su posición de mercado, y castigando al producto chileno.

El Gobierno de Chile, luego de gestiones diplomáticas y de su sector privado que no prosperaron, recurrió al Organo de Solución de Diferencias de la Organización Mundial del Comercio (OMC), basándose en el hecho de que esta regulación era una discriminación arbitraria que pretendía proteger al productor francés.

Aun cuando el caso se resolvió finalmente a través de una negociación directa que corrigió esta distorsión, la existencia de este mecanismo —obligatorio, independiente y vinculante- consagrado en la OMC promovió una solución satisfactoria que las gestiones diplomáticas de un país pequeño no lograron. Obviamente, éste no es el único ejemplo; se puede mencionar también el de las restricciones a las manzanas frescas impuestas por la Comunidad Económica Europea en 1993, y el de las restricciones a las exportaciones de tabaco impuestas por los Estados Unidos en 1993, ambos resueltos en el contexto del antiguo Acuerdo General sobre Aranceles Aduaneros y Comercio (GATT) ${ }^{5}$

\section{2. ¿Qué enseñan estas experiencias?}

En primer lugar, que el comercio internacional no tiene lugar en el vacío, sino que las posibilidades de aprovechar plenamente sus beneficios dependen no sólo de las políticas internas que adopte un país, sino

\footnotetext{
${ }^{5}$ Cabe reconocer que las soluciones alcanzadas no satisfacían ciento por ciento las aspiraciones chilenas, pero sí han permitido que estas actividades mantengan un adecuado desempeño económico.
}

que muy fundamentalmente de las que adopten los países con los cuales comercia.

En segundo lugar, que una de las principales funciones de los acuerdos internacionales es la de corregir fallas de mercado que la apertura unilateral no puede corregir. Con ellos se busca disminuir los costos de transacción que enfrentan los agentes privados al tomar decisiones de inversión. La literatura económica ha definido los costos de transacción como "los costos de medir el valor de los atributos de lo que se está intercambiando y los costos de proteger derechos y vigilar y hacer valer acuerdos". 6 Como señaló Coase (1988): sin 'el concepto de costos de transacción, que está ampliamente ausente de la actual teoría económica, es mi opinión que es imposible entender el funcionamiento del sistema económico, analizar muchos de sus problemas de manera útil, o tener una base para establecer políticas'.

Las relaciones económicas internacionales adolecen, entre otros problemas, de asimetrías de información; imperfecciones de mercado e incertidumbres (por ejemplo, desconocimiento de las condiciones de los mercados y de legislaciones que afecten el comercio y la estabilidad de los intercambios); falta de transparencia; y existencia de incentivos permanentes para desviarse de ciertos patrones de comportamiento (por ejemplo, a través de la proliferación de presiones proteccionistas). Todos ellos elevan los costos de operar en los mercados internacionales.

Los acuerdos cumplen dos funciones esenciales. En primer lugar, contribuyen a una apertura comercial recíproca que permite aprovechar las ganancias del comercio. En este contexto, la teoría de los juegos entrega los fundamentos económicos para concertar la apertura comercial entre países. En efecto, este caso es un ejemplo simple de la aplicación del dilema del prisionero: el mundo ideal para un país A es lograr que B desmantele sus barreras comerciales y poder mantener las propias; finalmente tanto A como B escogen mantener sus respectivas barreras, siendo éste el peor resultado para ambos en términos de bienestar. Las negociaciones comerciales de cualquier naturaleza abren oportunidades de cooperación permanentes una vez que los países han identificado sus estrategias conjuntas preferidas, pero para poder construir este espacio se necesitan mecanismos que identifiquen y castiguen el "fraude".

En segundo lugar, los acuerdos tienen la función de reducir los costos de transacción de las relaciones económicas internacionales, especialmente dada su multidimensionalidad actual, ya que proveen un con-

\footnotetext{
6 Véase otras definiciones en Dixit, 1996.
} 
junto de reglas aplicables a sus miembros que entregan estabilidad, estimulan la transparencia a través de obligaciones específicas y aseguran su cumplimiento mediante instrumentos vinculantes que reducen los incentivos para desviarse de las reglas. A esta última función Charles Kindleberger la ha denominado como la "provisión de servicios públicos de carácter internacional", por ser un "bien" necesario para la estabilidad de las transacciones comerciales que de no existir nos llevaría a la situación que se vivió en los años treinta en el mundo.

Vemos así que los acuerdos internacionales promueven el comercio a través de al menos dos mecanismos: i) la eliminación y reglamentación de las ba- rreras comerciales, proveyendo un marco de cooperación, y ii) la reducción de los costos de transacción. Ambos aspectos, así como los de carácter político, son normalmente ignorados en el debate público chileno sobre la racionalidad de la estrategia de los acuerdos comerciales. Como señala Coase (1988) es necesario 'introducir costos de transacción positivos explícitos en el análisis económico para que podamos estudiar el mundo que existe'.

Ahora bien, si existe la omc, que es una institución multilateral en la que participan más de 130 países, ¿cuál es el interés de negociar acuerdos económicos bilaterales? La respuesta a este interrogante tiene elementos económicos y por cierto también políticos.

\section{IV}

\section{El sistema multilateral de comercio}

El sistema multilateral de comercio representado por la omc es, desde el punto de vista de Chile, el foro de negociaciones económicas internacionales por excelencia, pues participan en él los principales países con los que Chile comercia. ${ }^{7}$ Desde luego, en este caso la opción de no ser miembro de la OMC no es una alternativa viable. Una negociación en este ámbito representa la mejor opción de Chile y la que brinda mayores beneficios por tener carácter multilateral, no involucrar desviación de comercio y reducir los costos de transacción con un conjunto de socios comerciales que operan bajo reglas comunes: Chile se beneficia de las concesiones económicas que se intercambian entre sus miembros, independientemente de su tamaño y peso relativo y del nivel de desarrollo que ostenta. Sin embargo, ésta también es una institución con diversas limitaciones e imperfecta. Algunas de sus imperfecciones se derivan de su propio carácter multilateral.

La última ronda de negociaciones multilaterales, y por cierto la más importante de este siglo, fue la denominada Ronda Uruguay del GATT (CEPAL, 1994). Esta ronda, que concluyó en diciembre de 1993, permitió que más de 120 países concluyeran un muy ambicioso conjunto de acuerdos que vinculan a Chile con el resto del mundo y dio un fuerte impulso a los

\footnotetext{
${ }^{7}$ La ausencia de la República Popular China y Rusia es un grave defecto que debe ser corregido con rapidez.
}

derechos y obligaciones que constituyen el sistema: en particular, no discriminación, trato nacional y cláusula de nación más favorecida.

Sin embargo, las negociaciones fueron muy complejas, las más largas de la historia del GATT, y no estuvieron exentas de grandes dificultades. De hecho, debieron haber concluido originalmente en diciembre de 1990. Sin embargo, no fue así, y entre 1990 y 1993 hubo gran incertidumbre respecto de qué ocurriría realmente con el sistema multilateral de comercio que se desarrolló, desde 1948, en torno del GatT. Académicos de diferentes orientaciones escribieron libros que hablaban de las guerras comerciales que vendrían entre los Estados Unidos, Japón y la Unión Europea (Thurow, 1991; Krugman, 1996 y Bhagwati, 1991).

Además, en este escenario muy incierto, Estados Unidos dio un giro significativo en su política comercial: emprendió una política activa de negociaciones comerciales bilaterales (Israel 1985, Canadá 1989 y NAFTA en 1992). La Unión Europea, dejando atrás el enfoque aplicado desde la Segunda Guerra Mundial, negoció y suscribió el Tratado de Maastricht (1992), el más importante desde el Tratado de Roma, y continuó con la incorporación de nuevos miembros (Frankel, 1997) en lo que algunos autores denominaron la "fortaleza europea". Es importante recordar que el Consejo Económico del Asia-Pacífico (APEC) se consolidó como foro económico regional para el AsiaPacífico en estos años, especialmente en 1993 (cuadros 1 y 2). Se ha estimado que un $90 \%$ de los miembros 


\begin{tabular}{|c|c|c|c|c|}
\hline & Africa/Medio Oriente & Europa & Las Américas & Asia-Pacífico \\
\hline 1957 & & 1957 Tratado de Roma. & & \\
\hline 1960 & & $\begin{array}{l}1960 \text { Asociación Europea. } \\
\text { de libre Comercio (AELC). }\end{array}$ & $\begin{array}{l}\text { Asociación Latinoamericana } \\
\text { de Libre Comercio (ALALC). }\end{array}$ & \\
\hline $\begin{array}{l}1965 \\
1970\end{array}$ & & & 1969 Pacto Andino. & Australia-Nueva Zelandia \\
\hline 1975 & $\begin{array}{l}\text { Comunidad Económica de } \\
\text { los Estados de Africa } \\
\text { Occidental. }\end{array}$ & & & $\begin{array}{l}1978 \text { Asociación de } \\
\text { Naciones del Sudeste } \\
\text { Asiático (ASEAN). }\end{array}$ \\
\hline 1980 & Conferencia Sudafricana. & & $\begin{array}{l}\text { Asociación Latinoamericana } \\
\text { de Integración (ALADI) }\end{array}$ & \\
\hline 1985 & $\begin{array}{l}1989 \text { Unión Arabe del } \\
\text { Maghreb. }\end{array}$ & & $\begin{array}{l}1988 \text { Acuerdo EE.UU.- } \\
\text { Canadá. }\end{array}$ & $\begin{array}{l}\text { 1985-1987 ASEAN se } \\
\text { se expande. }\end{array}$ \\
\hline 1990-1996 & $\begin{array}{l}1991 \text { Comunidad } \\
\text { Económica de Africa. }\end{array}$ & $\begin{array}{l}1991 \text { Area Económica } \\
\text { Europea (AELC+UE) } \\
1993 \text { Tratado de Maastricht. }\end{array}$ & $\begin{array}{l}1991 \text { Mercosur. } \\
1992 \text { NAFTA. } \\
1996 \text { Pacto Andino se } \\
\text { transforma en Comunidad } \\
\text { Andina. }\end{array}$ & $\begin{array}{l}1992 \text { Asociación Regional } \\
\text { del Asia Sudoriental. } \\
1993 \text { Consejo Económico } \\
\text { del Asia-Pacífico (APEC). }\end{array}$ \\
\hline
\end{tabular}

Fuente: Frankel (1997)

a Esta lista no es exhaustiva.

CUADRO 2

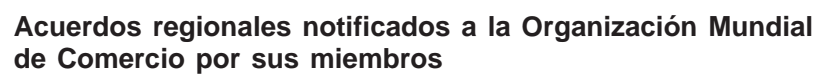

\begin{tabular}{cccccccccc}
\hline $1957-1990$ & 1991 & 1992 & 1993 & 1994 & 1995 & 1996 & 1997 \\
\hline 30 & 1 & 9 & 7 & 3 & 7 & $1991-1997$ & 20 & 61 \\
\hline
\end{tabular}

Fuente: OMC.

de la OMC pertenecen a algún tipo de acuerdo regional (Lawrence, 1996).

En América Latina, el Mercosur se transformó en la principal iniciativa de integración económica y la de mayor alcance, y ha tenido grandes efectos sobre el comercio hemisférico y el de Chile en particular, que por cierto van más allá de los aspectos exclusivamente económicos.

Los resultados de la Ronda Uruguay fueron muy significativos, reforzando y clarificando derechos y obligaciones en un número significativo de aspectos, logrando la incorporación de los sectores agrícola y de textiles y confecciones a las reglas multilaterales, y estableciendo el primer acuerdo multilateral sobre comercio de servicios y sobre aspectos de la propiedad intelectual relacionados con el comercio. La OMC ha suministrado un marco jurídico serio para la solución de diferencias. ${ }^{8}$ En efecto, desde la entrada en vigor de

\footnotetext{
${ }^{8}$ Véase entre otros Jackson (1997) y Petersman (1997).
}

la OMC y hasta el 27 de julio de 1998 se solicitó la realización de 141 consultas en el marco del Entendimiento sobre Solución de Diferencias. Sin embargo, cabe reconocer que no se lograron todos los avances que esperaban países como Chile — por ejemplo, en el acceso a mercados agrícolas o el desmantelamiento del Acuerdo Multifibras que distorsiona el comercio- y que existen aún espacios amplios para aplicar restricciones arbitrarias y encubiertas al comercio, así como vacíos en términos de disciplinas comerciales.

Así, la velocidad y profundidad de la apertura comercial que se puede lograr en el plano multilateral es limitada, aunque su fortalecimiento y profundización sigue siendo una prioridad para Chile.

En este panorama mundial de principios de los años noventa, en el que existía la posibilidad cierta de entrar en un período de "desorden" económico internacional, con conflictos entre las potencias económicas y la formación de bloques económicos excluyentes (el nAfTA, la Unión Europea, el Asia-Pacífico, el Mercosur, etc.), y se percibía la menor capacidad de 
liderazgo de los Estados Unidos en materia comercial, Chile buscó una forma de "proteger" y profundizar el esquema de desarrollo adoptado en las dos últimas décadas, caracterizado por su apertura económica y por un crecimiento estimulado por su comercio exterior. Esto, unido a consideraciones de política externa, explica que el país buscara acuerdos económicos internacionales como complemento de su política de apertura unilateral.

Así lo reconoció la propia OMC en su informe sobre la política comercial de Chile elaborado en septiembre de 1997, el que señala en parte: 'El actual interés de Chile por el regionalismo dimana de una clara determinación de no quedar excluido de los sistemas preferenciales que se están configurando, lo que lo llevaría a perder mercados, pero su intención proclamada de proceder a nuevas reducciones arancelarias unilaterales es prueba de que mantiene al mismo tiempo su compromiso de aplicar políticas de libre comercio basadas en el principio de nación más favorecida..., 9
Chile ha realizado los mayores esfuerzos, y ha buscado insistentemente oportunidades de negociación con aquellos países o grupos de países con los que más comercia (Estados Unidos, Mercosur, el Asia-Pacífico y la Unión Europea) y que son, asimismo, los que han emprendido procesos de integración con repercusiones económicas muy significativas; ${ }^{10}$ Chile ha tratado de asegurar y ampliar las oportunidades de negocios para su sector productivo y evitar marginarse o protegerse del comercio internacional.

Afortunadamente, los escenarios más pesimistas no se han dado, hasta el momento, en la realidad: en 1990-1996, el comercio internacional creció a una tasa media de $6 \%$ anual, en circunstancias que el PIB mundial lo hizo en sólo $1.5 \%$ y las exportaciones de Chile en $11 \%$. Además, estudios de la Organización de Cooperación y Desarrollo Económicos (OCDE) y de la propia OMC han reconocido que la proliferación de acuerdos regionales ha sido un complemento de la apertura multilateral o, al menos, no la han afectado negativamente. ${ }^{11}$

\section{$\mathrm{V}$}

\section{Los acuerdos bilaterales vigentes y sus efectos}

Para analizar los fundamentos que orientan la política comercial "lateral" que ha seguido Chile es pertinente abordar cuatro preguntas distintas pero relacionadas entre sí: ¿Qué tipo de acuerdos se debe negociar? ¿Con qué países se debe negociar? ¿Qué objetivos se han querido alcanzar? ¿Cuál es la evaluación de los acuerdos?

Al responder a estas preguntas, cabe señalar que los acuerdos que se han negociado no resuelven todos los problemas que se enfrentan. Tampoco son perfectos. En muchos aspectos han resultado insatisfactorios, lo que se ha querido subsanar con nuevas propuestas para mejorarlos y profundizarlos. Sin embargo, los acuerdos actuales de hecho han avanzado en la dirección de promover el libre comercio (cuadro 3 ).

\section{1. ¿Qué tipo de acuerdos se debe negociar?}

La teoría económica no responde de manera satisfactoria, o no ambigua, a esta pregunta. Dornbusch (1993)

\footnotetext{
${ }^{9}$ Véase el resumen y conclusiones, en омс (1997).
}

señala que: 'En el área de política comercial una buena dosis de sentido común debe llenar los vacíos dejados por la ausencia de una teoría sólida, la cual -de existir- debería proporcionar orientación'. En ese sentido, se dispone de criterios respecto a lo que no deben hacer los acuerdos comerciales: i) no deben desviar comercio (criterio expuesto ya por Jacob Viner en 1950), y ii) deben mantener el volumen de comercio de sus miembros respecto del resto del mundo y aumentar el volumen de comercio entre sus miembros

\footnotetext{
${ }^{10}$ En efecto, si bien las ganancias comerciales del ingreso de Chile al NAFTA o de una negociación bilateral han sido estimadas en cifras pequeñas, nunca se ha estimado el impacto de que países cuyas exportaciones compiten con las chilenas alcanzaran un acceso preferencial a ese mercado, como ocurre, desde luego, con los países que forman parte de la agrupación correspondiente y, también, con algunas preferencias que se otorgan en el marco del Sistema Generalizado de Preferencias (SGP) y a las que las exportaciones chilenas no tienen acceso. Algo similar podría ocurrir, asimismo, en el caso de un acuerdo entre el Mercosur y la Unión Europea y de ésta con Sudáfrica.

11 En parte debido a que en el marco del sistema multilateral de comercio se han producido rondas de negociaciones al menos una vez por década. Véase ocDE (1995) y OMC (1995).
} 
CUADRO 3

Chile: Estado de situación de los acuerdos bilaterales

\begin{tabular}{|c|c|c|}
\hline Acuerdo & Situación & Cobertura \\
\hline Con Canadá & $\begin{array}{l}\text { Vigencia: } 5 \text { de julio de } 1997 . \\
\text { Programas de desgravación en marcha según } \\
\text { previsto; quedará concluido el año } 2014 \text {. } \\
\text { Se prevé el inicio de negociaciones de servicios } \\
\text { financieros en } 1999 .\end{array}$ & Comercio de bienes y servicios e inversiones. \\
\hline $\begin{array}{l}\text { Con México } \\
\left(\mathrm{ACE} \mathrm{N}^{\mathrm{o}} 17\right)^{\mathrm{a}}\end{array}$ & $\begin{array}{l}\text { Vigencia: } 1 \text { de enero de } 1992 . \\
\text { Programa de desgravación completado. } \\
\text { Próximamente será enviado al Congreso Nacional } \\
\text { el nuevo tratado, que incorpora nuevas áreas. } \\
\text { Se prevé que en } 1999 \text { se iniciarán negociaciones } \\
\text { sobre servicios financieros, medidas antidumping y } \\
\text { compras de gobierno. }\end{array}$ & $\begin{array}{l}\text { Comercio de bienes. } \\
\text { Nuevo tratado: servicios, inversiones, propiedad } \\
\text { intelectual, obstáculos técnicos. } \\
\text { Medidas sanitarias y fitosanitarias. } \\
\text { Transporte aéreo. }\end{array}$ \\
\hline $\begin{array}{l}\text { Con Venezuela } \\
\left(\text { ACE } N^{\circ} 23\right)\end{array}$ & $\begin{array}{l}\text { Vigencia: } 1 \text { de julio de } 1993 . \\
\text { Programa de desgravación quedará concluido el } \\
1 \text { de enero de } 1999 .\end{array}$ & $\begin{array}{l}\text { Comercio de bienes. } \\
\text { Contempla compromiso de ampliación del } \\
\text { comercio de servicios. }\end{array}$ \\
\hline $\begin{array}{l}\text { Con Colombia } \\
\left(\mathrm{ACE} \mathrm{N}^{\circ} 24\right)\end{array}$ & $\begin{array}{l}\text { Vigencia: } 1 \text { de enero de } 1994 . \\
\text { Programa de desgravación quedará concluido el } \\
1 \text { de enero de } 1999 .\end{array}$ & $\begin{array}{l}\text { Comercio de bienes. } \\
\text { Se inició negociación para incorporar el comercio } \\
\text { de servicios e inversión. }\end{array}$ \\
\hline $\begin{array}{l}\text { Con Ecuador } \\
\left(\text { ACE } N^{\circ} 32\right)\end{array}$ & $\begin{array}{l}\text { Vigencia: } 1 \text { de enero de } 1995 . \\
\text { Programa de desgravación quedará concluido el } \\
1 \text { de enero del año } 2000 .\end{array}$ & $\begin{array}{l}\text { Comercio de bienes. } \\
\text { Contempla compromiso de ampliación del } \\
\text { comercio de servicios. }\end{array}$ \\
\hline $\begin{array}{l}\text { Con Perú } \\
\left(\text { ACE } N^{\circ} 38\right)\end{array}$ & $\begin{array}{l}\text { Vigencia: } 1 \text { de julio de } 1998 . \\
\text { Programa de desgravación en marcha, quedará } \\
\text { concluido el } 1 \text { enero del año } 2012 \text {. }\end{array}$ & $\begin{array}{l}\text { Comercio de bienes. } \\
\text { Contempla compromiso de ampliación del } \\
\text { comercio de servicios. }\end{array}$ \\
\hline $\begin{array}{l}\text { Con Mercosur } \\
\left(\mathrm{ACE} \mathrm{N}^{\circ} 35\right)\end{array}$ & $\begin{array}{l}\text { Vigencia: } 1 \text { de octubre de } 1996 . \\
\text { Programa de desgravación en marcha, quedará } \\
\text { concluido el } 1 \text { de enero del año } 2014 \text {. } \\
\text { Participación en estructura institucional del } \\
\text { Mercosur. }\end{array}$ & $\begin{array}{l}\text { Comercio de bienes. } \\
\text { Integración física. } \\
\text { Compromiso de negociación del comercio de } \\
\text { servicios. }\end{array}$ \\
\hline $\begin{array}{l}\text { Con Bolivia } \\
\left(\mathrm{ACE} \mathrm{N}^{\circ} 22\right)\end{array}$ & Vigencia: 6 de abril de 1993. & $\begin{array}{l}\text { Acuerdo de Alcance Parcial que incluye un número } \\
\text { específico de productos. } \\
\text { Se ha propuesto ampliar este acuerdo para } \\
\text { incorporar más productos en él. }\end{array}$ \\
\hline
\end{tabular}

a ACE: Acuerdos de complementación económica.

(teorema de Kemp y Wan), es decir, no deben crear barreras comerciales adicionales respecto de terceros países: esta regla asegura que los acuerdos se traduzcan en un aumento del bienestar social.

Un segundo aspecto, no abordado por la teoría económica, es cuál debe ser el contenido de la negociación. Cuando Viner (1950) escribió el trabajo en que distingue los efectos de creación y desviación de comercio (el cual ha servido de orientación para las investigaciones), los acuerdos a los que se refería eran fundamentalmente de tipo arancelario y, en menor medida, no arancelario (cuotas, licencias, contingentes, prohibiciones, etc.). En el contexto actual, en cambio, la agenda de negociaciones es mucho más amplia y compleja y se refiere a la necesidad de negociar "todos los aspectos que afectan el comercio". En este contexto, desde la Ronda Tokio del GATT, en 19741979, se observa una clara tendencia a abordar nuevos aspectos del comercio: barreras técnicas, compras de gobierno, derechos antidumping, subsidios y derechos compensatorios. El Tratado de Roma, el NAFTA y los resultados de la Ronda Uruguay fueron un paso adelante al incorporar temas nuevos como servicios, propiedad intelectual, y algunos aspectos de las inversiones. Menos exitosa ha sido la presión por incorporar cuestiones relacionadas con el medio ambiente y 
los derechos laborales, aunque esos temas están muy presentes en la actual agenda de comercio.

Chile ha reconocido esta multidimensionalidad, promoviendo la incorporación de la mayor parte de los aspectos relacionados con el comercio en las negociaciones que lleva adelante, pero lo ha hecho con una buena dosis de realismo. Así, el Tratado vigente con Canadá incorpora los más altos derechos y obligaciones existentes hasta hoy en acuerdos de libre comercio firmados por Chile en materia de servicios e inversiones; pero no incorpora aspectos como propiedad intelectual, normas técnicas, medidas sanitarias y fitosanitarias, compras de gobierno y otros, porque al negociar ambos países consideraron que las reglas de la OMC, junto a sus respectivas legislaciones, abordaban en forma satisfactoria estas materias.

Por otra parte, el Tratado que ha sido recientemente negociado con México sí los incorpora, porque en la relación bilateral con este país esas cuestiones son relevantes (los actuales problemas de acceso a México tienen que ver con materias de esta índole, como son las normas técnicas).

El realismo con que se ha enfocado esta estrategia también reconoce que no todos sus socios potenciales están interesados en ir más allá de lo logrado en la OMC respecto a la incorporación de todas las dimensiones del comercio, o simplemente no están en condiciones de hacerlo, por debilidades institucionales o de otra naturaleza.

\section{2. ¿Con qué países se debe negociar?}

La teoría económica ha sido incapaz de formular una regla o recomendación simple en términos de bienestar social respecto de qué países se debe escoger como socios para un acuerdo bilateral (Srinivasan, Whalley y Wooton, 1993). Por ejemplo, se considera que el socio principal de un país es un candidato natural: pero Chile cuenta con varios "socios principales", dependiendo de la agregación que se haga. También se señala que un país debe negociar con otros que tienen igual patrón de exportaciones o importaciones como una manera de modificar la relación de precios del intercambio en su favor, pero para Chile eso es muy poco factible. La geografía desempeña un papel muy importante en la elección de un socio comercial: la información empírica más reciente indica que dos países que mantienen una frontera común comercian un $82 \%$ más que dos países similares que no comparten límites. Asimismo, estas estimaciones muestran que un $1 \%$ de aumento en la distancia reduce en un $0.6 \%$ el comercio, obviamente siendo todo lo demás constante (Frankel, 1997).

La dimensión política, que es reconocida por la literatura económica como importante para explicar la formación de acuerdos regionales, en el caso de Chile no ha estado ausente. En efecto, el Canciller Insulza ha señalado que "los acuerdos de complementación e integración económica que hemos fomentado en los últimos años, aunque tienen un carácter comercial, pueden llegar a tener implicancias en términos de incrementar las condiciones de seguridad de Chile en nuestro entorno regional". Estas palabras también están respaldadas por los resultados de estudios empíricos que estiman las "externalidades de la seguridad": hay mayores niveles de comercio entre países que son aliados estratégicos que entre aquellos que se consideran o son adversarios (Mansfield, 1993).

La composición del comercio de Chile con América Latina, que favorece la exportación de bienes con mayor valor agregado, los servicios y la inversión, así como los vínculos culturales e históricos existentes (reconocidos asimismo en la literatura económica como determinantes del comercio) ${ }^{12}$ y la dimensión política anotada, explican la prioridad que Chile ha asignado a la región latinoamericana en su política comercial.

\section{3. ¿Qué objetivos se han querido alcanzar?}

\section{a) Acceso a mercados}

La política de negociación de acuerdos económicos internacionales se ha encaminado, en primer lugar, a abrir los mercados externos para asegurar el mejor desarrollo de las exportaciones de Chile. La apertura unilateral obviamente no tiene como contrapartida necesaria una apertura de los mercados de los socios comerciales de Chile. Por ejemplo, no tiene efectos en las prácticas aduaneras de éstos ni en la forma en que se adoptan y administran las barreras técnicas, y no eliminan las prácticas restrictivas que aplican los países mediante la adopción de medidas antidumping. En virtud de los acuerdos se puede lograr una apertura preferencial, segura y predecible que facilite el desarrollo de proyectos en el campo exportador.

b) Asegurar las condiciones de acceso y la estabilidad de las exportaciones

América Latina se ha caracterizado por condiciones económicas y políticas comerciales inestables. La

\footnotetext{
12 Véase las estimaciones contenidas en Frankel (1997).
} 
apertura comercial en la década de 1990 ha sido notable en todas las dimensiones del comercio. A través de los acuerdos bilaterales se han podido resguardar estas condiciones. Los tres ejemplos siguientes ilustran este punto. Desde 1997, Brasil, a raíz de sus dificultades financieras externas, ha venido aplicando medidas restrictivas a sus importaciones, extendiéndolas a Chile, Bolivia y al resto de sus socios del Mercosur; dos de estas medidas - la restricción de los créditos para el financiamiento de las importaciones y la aplicación de licencias no automáticas para el ingreso de importaciones - se han aplicado a Chile, Bolivia y el resto de los socios del Mercosur, con esquemas de operación excepcionales que, sin derogar las medidas, permiten una mantención parcial de las corrientes de comercio. Por su parte, México aumentó sus aranceles para los socios de la OMC después de 1993, pero no para Chile. Y Colombia, recientemente, quiso imponer restricciones a las exportaciones de algunos productos textiles de Chile en el marco de la OMC, pero en virtud del acuerdo bilateral esto no se hizo. Hay algunos casos, por ejemplo respecto del Mercosur, que aún no se han podido resolver satisfactoriamente, pero existe el marco de trabajo para hacerlo.

\section{c) Eliminar barreras al comercio que serían dífíciles de eliminar de otra manera}

El sistema multilateral de comercio tiene ciertas reglas de negociación que pueden inhibir la capacidad de negociar de un país pequeño como Chile. En efecto, la cláusula de nación más favorecida, que es un pilar del sistema por medio del cual Chile se ha beneficiado de aperturas negociadas por otros países, es también un elemento limitante en cierta medida: al no ser este país un productor importante a nivel mundial de un determinado bien, sus espacios de negociación son estrechos (regla del principal abastecedor y de interés sustancial).

Esto se puede ilustrar con un ejemplo concreto. En Chile existen actualmente tres compañías armadoras de automóviles: General Motors, Peugeot y Renault (esta última produce además algunas partes y piezas). El acuerdo Chile-México consagra la apertura de las exportaciones de vehículos hacia ese mercado en condiciones favorables, al margen de las disposiciones de su legislación automotriz: las reglas de origen son mucho más sencillas que las del NAFTA y, desde 1996, el comercio entre ambos países está completamente liberado. Las exportaciones chilenas de vehículos a México aumentaron de 7500 dólares en 1995 a 105000 en 1996, pasaron a 33 millones de dólares en
1997 y llegaron a 29 millones en el primer semestre de 1998. La eliminación de estas barreras no habría sido posible para Chile en una negociación multilateral, porque no es un productor relevante en el mercado mundial. La negociación bilateral estimuló una exportación que la apertura unilateral no había podido producir.

d) Avanzar en todas las dimensiones del comercio Las negociaciones de la Ronda Uruguay procuraron incorporar un número significativo de nuevas dimensiones a las reglas multilaterales vigentes desde la creación del GATT en 1947. Aun cuando los resultados fueron muy notables, se observan varias debilidades. La complejidad que se agrega a las negociaciones comerciales al incorporar más dimensiones y ser éstas sensibles, refuerza la idea de que existen mejores perspectivas bilaterales de resolver satisfactoriamente este tipo de dificultades.

En consecuencia, un primer objetivo es promover y proteger las inversiones de Chile en el exterior, las exportaciones de servicios que normalmente se asocian a éstas y las que no están asociadas a las inversiones. Así, por ejemplo, en el marco de estos acuerdos se ha podido avanzar y consolidar medidas para asegurar que los armadores chilenos accedan sin restricciones a las cargas bilaterales, así como a aquéllas desde y hacia terceros países en materia de transporte marítimo. Recientemente, en el marco de las negociaciones del nuevo Tratado con México, se eliminó la restricción indirecta sobre el transporte de vehículos que beneficiaba el transporte en buques mexicanos, otorgando así las mismas condiciones a chilenos y mexicanos. Esta controversia se había prolongado desde 1991.

Otro tema importante para Chile es la posibilidad de eliminar la aplicación de derechos antidumping. Este objetivo, muy difícil de alcanzar en el plano multilateral, se logró en la negociación con Canadá y se espera poder hacer lo mismo con México en un futuro próximo. Tales avances constituyen en efecto medidas ejemplares para las negociaciones comerciales hemisféricas en curso.

e) Proteger y estabilizar el acceso a los mercados La existencia de programas de preferencias unilaterales que discriminan contra algunas exportaciones; la implementación del NAFTA (o negociación de acuerdos bilaterales por parte de los Estados Unidos) en países cuyas exportaciones compiten con las de Chile en el mercado estadounidense; la negociación de acuerdos de la Unión Europea con el Mercosur o con Sudáfrica, y la formación misma del Mercosur, son hechos 
que pueden ser muy desfavorables para las exportaciones de Chile por los efectos de desviación de comercio y la pérdida de posición competitiva en esos mercados. Chile busca evitar tales efectos resguardando, al menos, las mismas condiciones de acceso de las que disfrutan sus competidores más directos.

\section{f) Promover la exportación de bienes manufactura- dos y de servicios}

La composición del comercio de Chile con los países latinoamericanos es distinta de la de su comercio con el resto del mundo. Las exportaciones chilenas a la región incluyen productos de mayor valor agregado y precios más estables, y los acuerdos bilaterales permiten aprovechar las economías de escala que ofrece un mercado más amplio e incorporar más empleo, tecnología e innovación, elementos centrales del desarrollo económico. Todo ello en la medida en que las reglas de origen sean simples y estén orientadas a la creación y no a la desviación de comercio.

Otra de las características de los vínculos de Chile con la región son las exportaciones de servicios. La complejidad de las negociaciones en este rubro se reduce significativamente en un medio en el que participa un menor número de países.

\section{4. ¿Cuál es la evaluación de los acuerdos?}

Para responder a esta pregunta se debe destacar, en primer lugar, que el acceso preferencial a mercados de casi 500 millones de habitantes ha redundado en el incremento y la diversificación de las exportaciones chilenas (cuadro 4).

Los acuerdos tienen lugar en entornos macroeconómicos y de economía internacional que son un dato de la realidad, pero cuyos efectos se busca amortiguar. Aun cuando el inicio de los años noventa se presentaba muy auspicioso en términos de crecimiento para los países de la región, desde 1994 éstos han experimentado diversos problemas que han afectado su desempeño macroeconómico. En efecto, cabe recordar el llamado "efecto tequila" de la crisis de México, los problemas de ajuste de las economías de Brasil y Argentina, y la inestabilidad política y económica prevaleciente en los últimos años en Colombia, Venezuela y Ecuador.

Esto, unido a la evolución del tipo de cambio bilateral de Chile y estos países, explica por una parte que Chile no haya podido aprovechar todas las nuevas opciones. Pero significa, por otra, que cuando se supere esa coyuntura, que no ha estado vinculada a la crisis asiática sino que a las reformas económicas

CUADRO 4

Chile: Comercio e inversión, por acuerdos suscritos y en agenda de negociación (Millones de dólares)

\begin{tabular}{|c|c|c|c|c|c|c|c|c|c|}
\hline Año & $\begin{array}{l}\text { Comunidad } \\
\text { Andina }^{\mathrm{a}}\end{array}$ & $\underset{\mathrm{b}}{\operatorname{Mercosur}}$ & México & Canadá & $\begin{array}{l}\text { Estados } \\
\text { Unidos }\end{array}$ & $\begin{array}{l}\text { Centro- } \\
\text { américa }^{\mathrm{c}}\end{array}$ & $\begin{array}{c}\text { Asia - } \\
\text { Pacífico }^{\mathrm{d}}\end{array}$ & $\begin{array}{l}\text { Unión } \\
\text { Europea }\end{array}$ & Totales \\
\hline \multicolumn{10}{|c|}{ Exportaciones de bienes } \\
\hline 1990 & 304.6 & 652.0 & 57.7 & 56.2 & 1469.2 & 12.9 & 2159.8 & 3279.8 & 8580.3 \\
\hline 1993 & 566.8 & 1089.2 & 130.8 & 61.1 & 1655.2 & 54.4 & 2839.7 & 2544.5 & 9416.2 \\
\hline 1997 & 1118.8 & 1863.1 & 376.3 & 131.0 & 2710.5 & 96.9 & 5629.0 & 4146.6 & 17024.8 \\
\hline \multicolumn{10}{|c|}{ Importaciones de bienes } \\
\hline 1990 & 506.3 & 1124.0 & 100.8 & 224.3 & 1373.4 & 4.4 & 915.4 & 1882.4 & 7023.4 \\
\hline 1993 & 454.7 & 1761.0 & 209.7 & 203.1 & 2477.4 & 19.4 & 1853.8 & 2312.3 & 10629.6 \\
\hline 1997 & 914.2 & 3193.2 & 1076.2 & 432.5 & 4332.6 & 79.7 & 2905.3 & 3957.0 & 18111.6 \\
\hline \multicolumn{10}{|c|}{ Inversión extranjera directa ${ }^{\mathrm{e}}$} \\
\hline 1990 & 1.5 & 6.1 & - & 252.9 & 270.9 & - & 56.7 & 328.7 & 1320.4 \\
\hline 1993 & 7.5 & 59.3 & 0.4 & 480.5 & 623.7 & - & 97.6 & 193.2 & 1729.8 \\
\hline 1997 & 26.6 & 94.7 & 9.9 & 678.8 & 913.4 & - & 181.8 & 2181.0 & 5041.2 \\
\hline \multicolumn{10}{|c|}{ Inversión chilena directa ${ }^{\mathrm{f}}$} \\
\hline 1990 & - & 13.9 & - & - & - & - & - & - & 15.9 \\
\hline 1993 & 55.3 & 616.1 & 2.5 & - & - & - & - & - & 2795.1 \\
\hline 1997 & 1014.7 & 3545.8 & 18.0 & - & 75 & - & 30 & - & 4730.9 \\
\hline
\end{tabular}

Fuente: Elaborado a base de estadísticas de la Dirección de Estudios de DIRECON.

a Chile tiene algún tipo de Acuerdo de Complementación Económica (ACE) con todos los países miembros de ella (véase el cuadro 3).

b Incluye cifras de Argentina, Brasil, Paraguay y Uruguay.

c Incluye cifras de Costa Rica, El Salvador, Guatemala, Honduras y Nicaragua

d No incluye economías del APEC, excepto Estados Unidos, Canadá y México.

e Materializada a través del D.L.600. No incluye cifras de Cap. XIV, mecanismo muy usado por países de la región latinoamericana.

f Detectada. 
emprendidas, el país contará con un marco preferencial que sabrá aprovechar.

La actual crisis asiática también da algunas indicaciones de cómo se ha comportado el comercio con los países de esa región. Las exportaciones a aquellos con los cuales hay acuerdos comerciales han crecido $8 \%$ en los primeros seis meses de 1998, siendo que las exportaciones totales al Asia-Pacífico han caído en un $11.4 \%$. Obviamente, el crecimiento señalado no puede atribuirse en $100 \%$ a la existencia de los acuerdos sino que, en gran medida, a la forma en que ha impactado la crisis y a la composición del comercio con estos socios. ${ }^{13}$ Pero el dinamismo de dicho comercio indica, de todos modos, la importancia de contar con una relación privilegiada en estos mercados.

Las evaluaciones de las acuerdos vigentes con el Mercosur, México, Colombia y Venezuela (ver Meller y Donoso, 1998, y Meller y Misraji, 1998), permiten ser optimistas. Si se evalúan los efectos de desviación y creación de comercio, criterio utilizado para medir el impacto de los acuerdos sobre el bienestar, los números indican que los primeros resultan muy pequeños, siendo los segundos muy significativos en algunos sectores. Como se señaló más atrás, este criterio es limitado, pues se concentra en los efectos de la eliminación de las barreras arancelarias y no toma en cuenta otras dimensiones del comercio incluidas en estos acuerdos. ${ }^{14}$ En particular, no puede medir la reducción de los costos de transacción que se derivan de la mayor certeza y transparencia de los regímenes comerciales vigentes en esos mercados, ni la expansión del comercio vinculada al proceso de "inversión hacia afuera".

Otro criterio utilizado por la teoría económica para evaluar los acuerdos comerciales (Kemp y Wan, 1976) es el siguiente: cuando el comercio entre los miembros de un acuerdo se expande y el de éstos con el resto del mundo se mantiene constante o se incrementa (no se crean obstáculos al comercio de terceros), existe un aumento de bienestar no ambiguo tanto para los miembros del acuerdo como para los no miembros. Si se mira lo que ocurría antes de la crisis asiática en el comercio de Chile con sus socios comerciales y con el resto del mundo, se observa que en términos globales el comercio chileno, tanto con aquellos países con los

\footnotetext{
13 Cabe recordar que son las exportaciones de cobre y del sector pesquero las que más inciden en la reducción de los montos exportados, por el efecto de la caída de los precios internacionales.

14 Tampoco considera cuestiones tales como la existencia de economías de escala (Pomfret, 1997).
}

que ha suscrito acuerdos como con el resto del mundo, ha seguido expandiéndose. ${ }^{15}$

En cuanto a la composición de las exportaciones chilenas, los estudios indican que los acuerdos han promovido, o bien resguardado, las exportaciones de bienes con mayor valor agregado. Esta ha sido una de las principales preocupaciones de los gobiernos de la Concertación. Pero el incremento de las exportaciones con mayor valor agregado también ha ido acompañado del aumento de las exportaciones tradicionales chilenas y de productos agrícolas que, en algunos casos, no se exportaban por diversas trabas que afectaban sus posibilidades de acceso a los mercados externos (por ejemplo, fitosanitarias).

La estimación del impacto económico de un acuerdo comercial es un ejercicio extremadamente complejo desde el punto de vista técnico y los resultados que se alcanzan dependen mucho de los supuestos con los que se elaboran.

Harrison, Rutherford y Tarr (1997) evalúan las distintas opciones de política comercial disponibles para Chile y su impacto en el bienestar social medido en términos de porcentaje anual del PIB (cuadro 5). Las opciones responden a una estrategia "aditiva", es decir, que da cuenta de cómo se afecta el bienestar social a medida que se van incorporando nuevos acuerdos. Las posibilidades evaluadas en el cuadro son: acuerdo con Mercosur; acuerdo con NAFTA; NAFTA más Mercosur; NAFTA más Mercosur y Unión Europea; NAFTA más Mercosur, Unión Europea y resto de Sudamérica, y por último, acuerdos con Canadá y México más Mercosur, Unión Europea y resto de Sudamérica. Además, los autores citados examinan el impacto que tienen estas opciones de política si se excluyen productos (por ejemplo, agrícolas sensibles) y si se rebaja el arancel de nación más favorecida desde 11 a $6 \%$.

Las principales conclusiones que emanan del cuadro 5 pueden resumirse así: la mejor opción es integrar en los acuerdos a todos los productos — es decir, que no existan exclusiones-, política que ha estado aplicando Chile. La mejor estrategia es suscribir acuerdos con el mayor número de socios a fin de evitar los costos provocados por la desviación de comercio; se puede decir que esto es un respaldo a la estrategia de regionalismo abierto defendida por el Gobierno de Chi-

\footnotetext{
15 Cabe reiterar que ésta es una conclusión respecto del conjunto del comercio; no pretende ser una generalización a nivel de sectores, en que la situación puede ser distinta. Véase una descripción de mecanismos de evaluación de acuerdos regionales en McMillan (1993).
} 


\begin{tabular}{|c|c|c|c|c|c|c|}
\hline \multirow[b]{2}{*}{ Cobertura de productos } & \multicolumn{6}{|c|}{$\begin{array}{l}\text { Chile: Una estimación de las ganancias de bienestar } \\
\text { derivadas de acuerdos comerciales } \\
\text { (Porcentajes del PIB) }\end{array}$} \\
\hline & Mercosur & Nafta & $\begin{array}{c}\text { Nafta } \\
\text { y Mercosur }\end{array}$ & $\begin{array}{l}\text { Nafta, Mercosur } \\
\text { y Unión Europea }\end{array}$ & $\begin{array}{c}\text { Nafta, Mercosur, } \\
\text { Unión Europea } \\
\text { y resto de } \\
\text { Sudamérica }\end{array}$ & $\begin{array}{c}\text { Canadá y México, } \\
\text { Mercosur, } \\
\text { Unión Europea y } \\
\text { resto de Sudamérica }\end{array}$ \\
\hline Sin excepciones & -0.43 & 1.04 & 1.48 & 5.24 & 8.4 & 8.16 \\
\hline Con excepciones & -0.43 & 1.04 & 1.48 & 2.02 & 2.48 & 0.44 \\
\hline Con excepciones y arancel de $6 \%$ & 0.35 & 1.70 & 2.01 & 2.29 & 2.66 & 0.87 \\
\hline $\begin{array}{l}\text { Sólo exclusión de prod. agrícolas } \\
\text { en acuerdo con la Unión Europea } \\
\text { Sólo exclusión de prod. agrícolas } \\
\text { en acuerdo con la Unión Europea }\end{array}$ & -0.43 & 1.04 & 1.48 & 2.02 & 5.48 & 3.90 \\
\hline y $6 \%$ de arancel & 0.35 & 1.70 & 2.01 & 2.29 & 5.71 & 4.44 \\
\hline
\end{tabular}

Fuente: Harrison, Rutherford y Tarr (1997).

le. Por otra parte, en un escenario como el descrito, excluir a los Estados Unidos no tiene un impacto significativo en el bienestar. Sin embargo, si se considera la posibilidad de excluir productos, la presencia o ausencia de los Estados Unidos es muy significativa. Finalmente, el cuadro indica que si sólo el acuerdo con

\section{VI}

\section{Conclusiones}

En este artículo se han examinado los fundamentos, los objetivos y los resultados de la política comercial "lateral" adoptada por Chile a partir de la década de 1990. Las críticas que se han formulado a esta estrategia se basan en un análisis limitado de los fundamentos teóricos del comercio internacional. Aquí se argumenta que para evaluar la racionalidad económica y política de esta estrategia es importante ampliar los conceptos económicos, reconociendo la complementariedad que existe entre las opciones disponibles e incorporando el análisis de la teoría de los juegos, la existencia de economías de escala, los costos de tran- la Unión Europea excluye productos agrícolas, el efecto en el bienestar es mayor que en la opción en la cual todos los acuerdos excluyen este tipo de productos, por la importancia que en un acuerdo bilateral con los Estados Unidos tienen para Chile los productos agrícolas no cereales. sacción que se enfrentan en el funcionamiento de los mercados internacionales, y también elementos de política exterior.

Esta estrategia multidimensional ha tenido como objetivo superar los problemas anotados anteriormente y estimular las actividades económicas más dinámicas que han caracterizado a la economía chilena durante los años noventa: las exportaciones con mayor valor agregado, de servicios y de capitales.

Si se aplican parámetros tradicionales de evaluación, los resultados obtenidos no acusan efectos negativos y sí se observan efectos positivos.

\section{Bibliografía}

Bhagwati, J. (1991): The World Trading System at Risk, Princeton, New Jersey, Princeton University Press.

CEPAL (Comisión Económica para América Latina y el Caribe) (1994): La Ronda Uruguay de Negociaciones Comerciales: una evaluación para América Latina y el Caribe, Santiago de Chile, septiembre.

Coase, R.H. (1988): The Firm, the Market and the Law, Chicago, Illinois, The University of Chicago Press. 
Dixit, A. (1996): The Making of Economic Policy: A TransactionCost Politics Perspective, Cambridge, Massachusetts, The MIT Press.

Dornbusch, R. (1993): The case for bilateralism, D. Salvatore (ed.), Protectionism and World Welfare, Londres, Cambridge University Press.

El Mercurio (1998): Santiago de Chile, Empresa Periodística El Mercurio, S.A.P., 14 de julio.

Frankel, J. (1997): Regional Trading Blocs, Washington, D.C., Institute for International Economics (IIE).

Harrison, G.W., T.F. Rutherford y D. G. Tarr (1997): NAFTA, MERCOSUR, and additive regionalism in Chile: A quantitative evaluation, Washington, D.C

Jackson, J.H. (1997): The World Trading System, Cambridge, Massachusetts, The MIT Press.

Kemp, M. y H.Y. Wan (1976): An elementary proposition concerning the formation of customs unions, Journal of International Economics, vol. 6, $\mathrm{N}^{\circ}$ 1, Amsterdam, Países Bajos, NorthHolland Publishing Company.

Krugman, P. (1996): Pop Internationalism, Cambridge, Massachussets, The MIT Press.

Lawrence R. (1996): Regionalism, Multilateralism, and Deeper Integration, Washington, D.C., The Brookings Institution.

Mansfield, E.D. (1993): Effects of international politics on regionalism in international trade, K. Anderson y R. Blackhurst (eds.), Regional Integration and the Global Trading System, Ginebra, Suiza, Harvester Wheastsheaf.

McMillan, J. (1993): Does regional integration foster open trade? Economic theory and GATT's Article XXIV, K. Anderson y R. Blackhurst (eds.), Regional Integration and the Global Trading System, Ginebra, Suiza, Harvester Wheastsheaf.

Meller, P (1996): Un siglo de economía política chilena, Santiago de Chile, Editorial Andrés Bello.

Meller, P. y R. Donoso (1998): La industria chilena y el Mercosur, Santiago de Chile, Dolmen Ediciones.
Meller, P. y D. Misraji (1998): Chile y los ALC con México, Colombia y Venezuela, Santiago de Chile, Universidad de Chile, Facultad de Ciencias Físicas y Matemáticas, Departamento de Ingeniería Industrial, enero.

OCDE (Organización de Cooperación y Desarrollo Económicos) (1995): Regional Integration and the Multilateral Trading System, París.

OMC (Organización Mundial del Comercio) (1995): El regionalismo y el sistema mundial de comercio, Ginebra.

(1997): Trade Policy Review: Chile 1997, Ginebra.

Petersman, E.U. (1997): International Trade Law and the GATT/WTO Dispute Settlement System, La Haya, Luwer Law International.

Pomfret, R. (1997): The Economics of Regional Trading Arrangements, Oxford, Clarendon Press.

Sáez, R. (1994a): La Corporación de Fomento de la Producción y la diversificación industrial de Chile, Hombre del siglo $X X$, Santiago de Chile, Dolmen Ediciones

(1994b): La Corporación de Fomento de la Producción a cuarenta años de su creación, Hombre del siglo $X X$, Santiago de Chile, Dolmen Ediciones.

Sáez, S., J. Salazar y R. Vicuña (1995): Antecedentes y resultados de la estrategia comercial del Gobierno Aylwin, Estudios CIEPLAN, $\mathrm{N}^{\circ}$ 41, Santiago de Chile, Corporación de Investigaciones Económicas para Latinoamérica (CIEPLAN).

Srinivasan, T.N., J. Whalley e I. Wooton (1993): Measuring the effects of regionalism on trade and welfare, K. Anderson y R. Blackhurst (eds.), Regional Integration and the Global Trading System, Ginebra, Suiza, Harvester Wheastsheaf.

Thurow, L. (1991): Head to Head, Princeton, New Jersey, Princeton University Press.

Viner, J. (1950): The Custom Union Issue, Nueva York, Carnegie Endowment for International Peace.

Wisecarver, D. L. (ed.) (1992): El modelo económico chileno, Santiago de Chile, Centro Internacional para el Desarrollo Económico (CINDE)/Pontificia Universidad Católica de Chile, Instituto de Economía. 